\title{
Characteristics of Liposarcoma Patients: 5-Year Data
}

\author{
Miftahur Rahmah, ${ }^{1}$ Afiati, ${ }^{2}$ Unwati Sugiri ${ }^{3}$ \\ ${ }^{1}$ Faculty of Medicine Universitas Padjadjaran, ${ }^{2}$ Department of Anatomical Pathology Faculty of \\ Medicine Universitas Padjadjaran/Dr. Hasan Sadikin General Hospital Bandung, ${ }^{3}$ Department \\ of Dermatovenereology Faculty of Medicine Universitas Padjadjaran/Dr. Hasan Sadikin General \\ Hospital Bandung, Indonesia
}

\section{Abstract}

Background: Liposarcoma is the second highest soft tissue sarcoma in adults with prevalence of $15 \%$ to $25 \%$. If the size of liposarcoma is very large, it may cause abdominal pain, weight loss, gastrointestinal bleeding, even kidney failure. Therefore, for early detection of liposarcoma, the characteristics of liposarcoma should be investigated. This study aimed to determine liposarcoma characteristics based on age, sex, location and histopathologic type according to WHO (World Health Organization) Classification of Tumors 2013.

Methods: A descriptive study was conducted from August to October 2015 at the Department of Anatomic Pathology, Dr. Hasan Sadikin General Hospital, Bandung, Indonesia. Data was obtained from database of patients with liposarcoma at the Department of Anatomic Pathology in the period of January 2010 to December 2014. The collected data was presented in the form of figures and tables.

Results: Out of 102 cases, the percentage of liposarcoma was highest between the age of 50 and 59 years $(31.37 \%)$, followed by the age of $40-49$ years $(28.43 \%)$. The percentage were slightly higher in males (54.9\%) compared to females with a ratio of $1.22: 1$. Femur $(23.52 \%)$, intraabdomen $(22.55 \%)$ and retroperitoneum $(11.76 \%)$ were the most common locations affected. The most common histopathologic subtype was myxoid liposarcoma (52.95\%) exceeding a half of the total cases.

Conclusions: Liposarcoma most likely occurs in the age range of 40-60 years, the percentage is slightly higher in males, and abdomen and lower extremities are the most common location affected. Myxoid liposarcoma is the most common subtype.

Keywords: Liposarcoma, lower extremities, myxoid liposarcoma

\section{Introduction}

Liposarcoma is a malignant tumor that consists of differentiated fat cells and the second highest soft tissue sarcoma in adults. ${ }^{1,2}$ Prevalence of liposarcoma reached $15 \%$ to $25 \%$ of all sarcomas. ${ }^{3}$ Histologically, the World Health Organization (WHO) classifies liposarcoma into five subtypes, namely atypical lipomatous tumor/well differentiated, dedifferentiated, myxoid, pleomorphic and liposarcoma not otherwise specified. ${ }^{4}$

Clinically, liposarcoma is difficult to be diagnosed because it grows slowly and silently with no typical symptoms. ${ }^{1}$ Hence, a pathology examination is very important to diagnose liposarcoma. Liposarcoma usually occurs in the retroperitoneum and extremities. ${ }^{1,5-8}$ Retroperitoneal liposarcomas are difficult to treat and have higher rates of local recurrence, moreover it may cause death from uncontrolled local effects. ${ }^{6,9}$ If the tumor is very large, it may cause abdominal discomfort, abdominal pain, weight loss, gastrointestinal bleeding, even kidney failure., ${ }^{2,10-12}$ The only effective treatment for retroperitoneal liposarcoma is complete surgical resection. . $^{6,9,13-16}$

Data relating to age and sex are helpful for early detection of liposarcoma, meanwhile data about location and histopathological type of liposarcoma is important to predict tumor's prognosis. ${ }^{1,2,9}$ Unfortunately, there is no data about liposarcoma's characteristics at Dr. Hasan Sadikin General Hospital Bandung, Indonesia. Therefore, the objectives of this study were to identify the characteristics of liposarcoma based on age, sex, location and histopathological findings according to WHO Classifications of Tumors 2013.

Correspondence: Miftahur Rahmah, Faculty of Medicine, Universitas Padjadjaran, Jalan Raya Bandung-Sumedang Km.21, Jatinangor, Sumedang, Indonesia, Email: amahmiftah95@gmail.com 


\section{Methods}

This descriptive study was conducted from August to October 2015 at the Department of Anatomic Pathology, Dr. Hasan Sadikin General Hospital, Bandung. Ethical clearance was approved by the Health Research Ethics Committees of the Faculty of Medicine Universitas Padjadjaran.

The study data were obtained retrospectively by total sampling method from the database of patients with liposarcoma in the Department of Anatomic Pathology, Dr. Hasan Sadikin General Hospital during the period of January 2010 to December 2014. The variables collected in this study were patient age, sex, tumor location, and histopathological type of tumor according to WHO Classifications of Tumors 2013 (Atypical lipomatous tumor, dedifferentiated, myxoid, pleomorphic and liposarcoma not otherwise specified). ${ }^{4}$ Patients with incomplete data were excluded from the study. The collected data were presented in the form of frequency and proportion (percentage).

\section{Results}

A total of 102 cases of liposarcoma were collected in this study. The percentage of cases increased from year 2011 to 2014 (Figure 1). The percentage of cases was slightly higher on males compared to females with a ratio of 1.22:1. The highest percentage was between the age of 50-59 years, followed by the age of 40-49 years, whereas the youngest patient was 18 years old and the oldest was 78 years old.

Most of the liposarcoma location was in the abdomen, followed in the lower extremities. Liposarcoma found in the abdomen was mostly located in the intra abdomen (23 cases), followed in the retro peritoneal (12 cases), kidney (2 cases), and abdominal wall (one case). Moreover, Liposarcoma found in the upper extremities, was mostly located in the femur (24 cases), gluteus ( 7 cases), cruris ( 2 cases), pedis ( 2 cases), and popliteal (one case). The neck was the rarest affected location. In this study, the most common histopathological type that occured was myxoid liposarcoma (52.95\%), exceeding a half of the total cases (Table).

\section{Discussion}

Liposarcoma is a malignant tumor that consists of differentiated fat cells, and is the second highest soft tissue sarcoma in adults with prevalence reached $15 \%$ to $25 \%$ of all sarcomas.1-3 In this study, the precentage of liposarcoma increased from 2011 to 2014.

According to several literatures, liposarcoma is commonly found between the age of 40 and 60 years with slightly male predominance, although the precentage of retro peritoneal liposarcoma is higher in females. $1,2,5,8,17$ This is similar with this study which revealed that the highest percentage of liposarcoma are between the age of 50 and 59 years. The percentage of liposarcoma in this study was slightly higher in males than females with a ratio of 1.22:1. However, a study in Japan stated that liposarcoma affected both sexes equally.

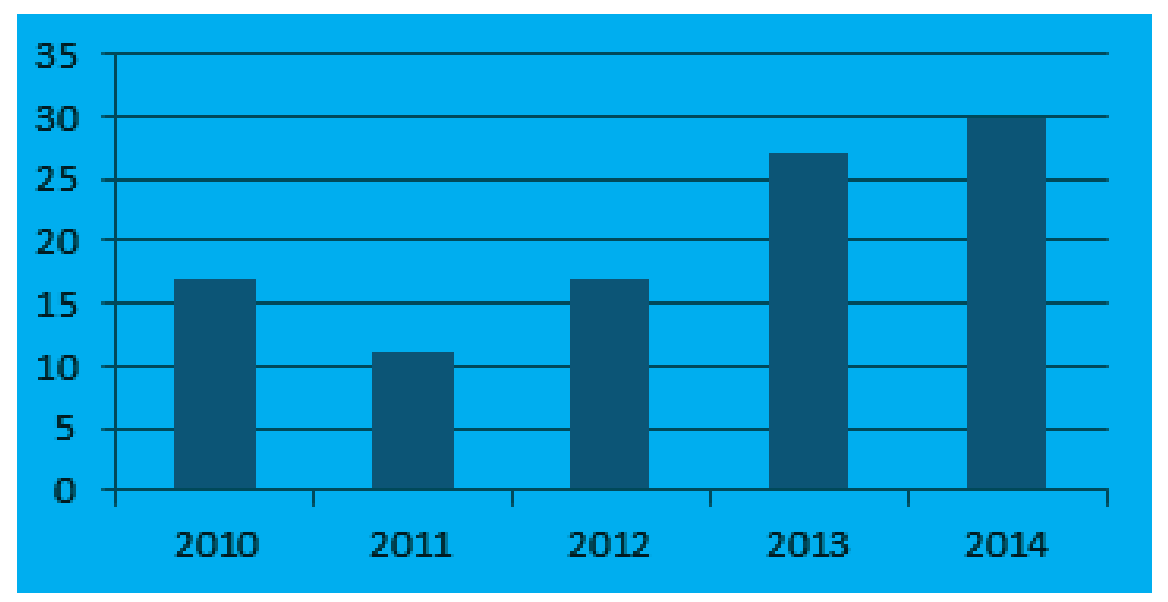

Figure Percentage of Liposarcoma Cases at Dr. Hasan Sadikin General Hospital, Bandung, in $2010-2014$ 
Table Characteristics of Liposarcoma's Patients Based on Age, Sex, Location and Histopathological Findings

\begin{tabular}{|c|c|c|}
\hline Variable & $n=102$ & $\%$ \\
\hline \multicolumn{3}{|l|}{ Sex } \\
\hline Male & 56 & $54.9 \%$ \\
\hline Female & 46 & $45.1 \%$ \\
\hline Age (year) & $56.6 \pm 9.7$ & $56.6 \pm 9.7$ \\
\hline $10-19$ & 1 & $0.98 \%$ \\
\hline $20-29$ & 6 & $5.88 \%$ \\
\hline $30-39$ & 12 & $11.77 \%$ \\
\hline $40-49$ & 29 & $28.43 \%$ \\
\hline $50-59$ & 32 & $31.37 \%$ \\
\hline $60-69$ & 18 & $17.65 \%$ \\
\hline $70-79$ & 4 & $3.92 \%$ \\
\hline Location & 20 & 27.8 \\
\hline Head & 7 & $6.86 \%$ \\
\hline Neck & 2 & $1.96 \%$ \\
\hline Trunk & 3 & $2.94 \%$ \\
\hline Abdomen & 38 & $37.26 \%$ \\
\hline Pelvis and perineum & 5 & $4.90 \%$ \\
\hline Upper extremities & 11 & $10.78 \%$ \\
\hline Lower extremities & 36 & $35.30 \%$ \\
\hline \multicolumn{3}{|l|}{ Histopathologic Feature } \\
\hline Atypical lipomatoustumors & 27 & $26.47 \%$ \\
\hline Dedifferentiated liposarcoma & 6 & $5.88 \%$ \\
\hline Myxoid liposarcoma & 54 & $52.95 \%$ \\
\hline Pleomorphic liposarcoma & 8 & $7.84 \%$ \\
\hline Liposarcoma not otherwise specified & 7 & $6.86 \%$ \\
\hline
\end{tabular}

Liposarcoma usually arises from deepseated, well-vascularized structures, and rarely from submucosal or subcutaneous fat., ${ }^{2,4}$ Abdomen and proximal extremities are the most common locations affected but some studies in South Korea, Japan and New Delhi reported that liposarcoma may also arise in the mesentery and abdominal wall. ${ }^{1,2,7-9,16-18}$ The percentage of liposarcoma reached 24\% of extremity sarcoma and occurred in the lower extremities four times more than in the upper extremities and reached $41 \%$ of retroperitoneal sarcoma. ${ }^{2,9}$ This was similar to this study in which the femur, intraabdomen, and retro peritoneum were the most common locations affected by liposarcoma.
The World Heatlh Organization classifies liposarcoma into atypical lipomatous tumors, dedifferentiated liposarcoma, myxoid liposarcoma, pleomorphic liposarcoma and liposarcoma not otherwise specified. $^{4}$ This histologic diversity influences biologic behaviour and prognosis of liposarcoma. ${ }^{1,2}$ Atypical lipomatous tumors are classified as intermediate or locally aggressive tumors because they grow slowly and do not metastasize, whereas the other types are malignant. ${ }^{4}$ The pleomorphic liposarcoma is a high-grade tumor and often metastasize accounting for the decreased rate of survival, whereas myxoid liposarcoma is a low-grade tumor which grows slowly. ${ }^{9,16}$ Dedifferentiated 
liposarcoma contains an abrupt transition between atypical lipomatous tumors and highgrade nonlipogenic sarcoma, histologically. Approximately $40 \%$ of dedifferentiated liposarcoma will recur locally in 10-20 years after therapy, $17 \%$ will metastasize, and $28 \%$ of patients will die as a result of this tumor.,9

According to WHO Classification of Tumors 2013, the largest subgroup of liposarcoma which accounts for $40-45 \%$ of all liposarcoma is atypical lipomatous tumors whereas, the rarest subtype is pleomorphic liposarcoma which accounts for $5 \%$ of all liposarcomas. ${ }^{2,5,16}$ A study from the Memorial Sloan-Kettering Cancer Center also stated that the mostcommon subtype of liposarcoma are atypical lipomatous tumors and dedifferentiated liposarcoma. ${ }^{9}$ On the contrary, myxoid liposarcoma was the most common subtype that occured in this study (52.43\%), and was double the atypical lipomatous tumors, whereas the rarest subtype was dedifferentiated liposarcoma which only accounted for $5.82 \%$ of all cases. This was similar to a study in New Delhi which stated that myxoid liposarcoma is the most common type of liposarcoma, and is found in 50\% cases. ${ }^{18}$ Another study in Tunisia also stated that myxoid liposarcoma is the second most common type of liposarcoma and represents 20 to $50 \%$ of all liposarcomas. ${ }^{2}$ Different study locations may have impacts on the differences of the largest and the rarest subtype in each country. The cause of the high percentage of myxoid liposarcoma at Dr. Hasan Sadikin General Hospital, Bandung was unknown, and requires a further study.

The limitation of this study was that there was some missing data in 2010 , so that it could not be recorded and processed.

The conclusion of this study is liposarcoma most likely occurs between the ages of 40 and 60 years. The incidence is slightly higher in males, which occurs most commonly in the femur, intraabdomen, and retroperitoneum with myxoid liposarcoma as the most common subtype occurrence

\section{References}

1. Lu J, Qin $Q$, Zhan L, Yang X, Xu Q Yu J, et al. Computed tomography manifestations of histologic subtypes of retroperitoneal liposarcoma.Asian Pac J Cancer Prev. 2014;15(15):6041-6.

2. Ouni FE, Jemni $H$, Trabelsi A, Maitig MB, Arifa N,Rhouma KB, et al. Liposarcoma of the extremities: MR imaging features and their correlation with pathologic data. Orthop Traumatol Surg Res. 2010;96(8):876-83.

3. Goldblum JR, Folpe AL,Weiss SW. Enzinger and Weiss's soft tissue tumors. 6th ed. Philadelphia: Saunders; 2014. p. 443-521.

4. Fletcher CDM, Bridge JA, Hogendoorn PCW, Mertens F. WHO classification of tumours of soft tissue and bone. 4th ed. Lyon: IARC; 2013. p. 19-44.

5. Tsuruta A, Notohara K, Park T, Itoh T. Dedifferentiated liposarcoma of the rectum: a case report. World J Gastroenterol. 2012;18(41):5979-81.

6. Lu W, Lau J, Xu MD, Zhang Y, Jiang Y, Tong HX, et al. Recurrent abdominal liposarcoma: analysis of 19 cases and prognostic factors. World J Gastroenterol. 2013;19(25):404552.

7. Cha EJ. Dedifferentiated liposarcoma of the small bowel mesentery presenting as a submucosal mass. World J Gastrointest Oncol. 2011;3(7):116-8.

8. Takeda $\mathrm{K}$, Aimoto $\mathrm{T}$, Yoshioka $\mathrm{M}$, Nakamura Y, Yamahatsu K, Ishiwata T, et al. Dedifferentiated liposarcoma arising from the mesocolon ascendens : report of a case. J.Nippon Med Sch. 2012;79(5):385-90.

9. Crago AM, Singer S. Clinical and molecular approaches to well-differentiated and dedifferentiated liposarcoma. Curr Opin Oncol. 2011;23(4):373-8.

10. Perez-Ponce Y, Castellanos-Alejandre $\mathrm{R}$, Guerrero-Romero JF, Estrada-Leon F, Torres-Lobaton A. Retroperitoneal liposarcoma as etiology of abdominal pain. Case report and literature review. Cir Cir. 2008;76(1):77-82.

11. Wanchick K, Lucha P. Dedifferentiated retroperitoneal liposarcoma presenting as lower gastrointestinal bleeding, a case report and review of the literature. Mil Med. 2009;174(3):328-30.

12. Gupta A, Pacha O, Skaria R, Huynh T, Truong L, Abdellatif A. Retroperitoneal sarcoma presenting as acute renal failure, secondary to bilateral renal artery invasion. Clin Nephrol. 2012;78(2):164-8

13. Petronella P, Scorzelli M, Lannacci G, Feretti M, Fiore A, Freda F, et al. Clinical considerations on the retroperitoneal liposarcomas. Ann ItalChir. 2012;83(1):359.

14. Na JC, Choi KH, Yang SC, Han WK. Surgical experience with retroperitoneal liposarcoma in a single Korean tertiary medical center. Korean J Urol. 2012;53(5):310-6.

15. Milone M, Pezzullo LS, Salvatore G, 
Pezzullo MG, Leongito M, Esposito FMI. Management of high-grade retroperitoneal liposarcomas: personal experience. Updates Surg. 2011;63(2):119-24.

16. Lee SY, Goh BKP, Teo MCC, Chew MH, Chow $\mathrm{PKH}$, Wong WK, et al. Retroperitoneal liposarcomas: the experience of a tertiary Asian center. World J Surg Oncol. 2011;9(1):1-6.
17. Kumar V, Abbas AK, Fausto N, Aster JC. Robbin and Cotran Pathologic Basis of Disease. $8^{\text {th }}$ ed. Philadelphia: Saunders; 2010. p. 1248-50.

18. Jain SK, Mitra A, Kaza RC, Malagi S. Primary mesentericliposarcoma: an unusual presentation of a rare condition. J GastrointestOncol. 2012;3(2):147-50. 